\title{
Ratmoko, Christina, Damit die Chemie stimmt. Die Anfänge der industriellen Herstellung von weiblichen und männlichen Sexualhormonen 1914-1938
}

Paul Pasteur

\section{OpenEdition}

Édition électronique

URL : http://journals.openedition.org/ifha/6702

DOI : $10.4000 /$ ifha.6702

ISSN : 2198-8943

Éditeur

IFRA - Institut franco-allemand (sciences historiques et sociales)

\section{Référence électronique}

Paul Pasteur, «Ratmoko, Christina, Damit die Chemie stimmt. Die Anfänge der industriellen Herstellung von weiblichen und männlichen Sexualhormonen 1974-1938 », Revue de l'IFHA [En ligne], Date de recension, mis en ligne le 01 janvier 2011, consulté le 22 septembre 2020. URL : http:// journals.openedition.org/ifha/6702 ; DOI : https://doi.org/10.4000/ifha.6702

Ce document a été généré automatiquement le 22 septembre 2020.

(CIFHA 


\title{
Ratmoko, Christina, Damit die Chemie stimmt. Die Anfänge der industriellen Herstellung von weiblichen und männlichen Sexualhormonen 1914-1938
}

\author{
Paul Pasteur
}

1 Cet ouvrage qui reprend la thèse soutenue par C.R. en 2008, explore les débuts de la production d'hormones ainsi que le processus qui a conduit à leur production industrielle à travers les hésitations, les rectifications de dosage et de prescription.

2 En 1905, le physiologue anglais Ernest Starling introduit le concept d'hormone. C.R. rappelle que, dès le départ, aussi bien le discours des scientifiques que celui des vulgarisateurs et plus encore le discours populaire tiennent à donner un genre à ces hormones. Bien que depuis les années trente, le genre de ces hormones ait été fondamentalement remis en question, ces substances sont encore décrites comme masculines ou féminines, ainsi les hormones " masculines " sont présentées comme permettant le rajeunissement et l'accroissement de la puissance sexuelle. Les hormones "féminines ", œstrogènes et progestérone, sont utilisées quotidiennement par plus de soixante dix millions de femmes sous la forme de la pilule. Mais lorsque l'auteure commence son récit, la recherche n'en est qu'à son début et il faut attendre trente ans pour que les cinq principales hormones (œstrone, œstradiol, progestérone, androstérone, testostérone) soient isolées, synthétisées puis distribuées. Néanmoins, dès le début du XXe siècle, l'industrie chimique produit et diffuse des extraits d'hormones animales qui sont vendus comme médicaments et l'auteure pose la question de la frontière entre thérapie et consommation, car elle montre bien que pendant les trois décennies décrites, l'entreprise, pour ne pas dire les marchés, font pression sur les chercheurs pour qu'ils livrent des produits rapidement commercialisables.

3 À partir des archives de la Ciba (Gesellschaft für Chemische Industrie) de Bâle, du fonds de Leopold Ruzicka qui a été à l'École polytechnique de Zürich, des publications des 
chercheurs et des entreprises, C.R. suit pas à pas les découvertes, l'élaboration et la diffusion de ces hormones sexuelles qui sont devenues des produits de consommation industrielle. Toutes ne parviennent pas à terme suivant les mêmes modalités, certains chemins sont tortueux, d'autres bénéficient de collaboration avec des médecins ou gynécologues allemands ou autrichiens. L'ouvrage se divise en cinq chapitres précédés d'une introduction et suivis d'une conclusion. Dans le premier chapitre, l'auteure revient sur les débuts de la Ciba spécialisée dans le goudron et qui ne commence à s'intéresser aux médicaments que dans les années 1880. Le second chapitre se concentre sur l'agomensin et le sistomensin qui ont bénéficié des découvertes des gynécologues et des médecins. Dans un troisième temps, C.R. revient sur la restructuration de l'entreprise qui élargit son département de pharmacologie et met en place un département de communication. Le quatrième chapitre est consacré au prokliman et à l'androstin pour les hommes, mis sur le marché au milieu des années trente. Le dernier chapitre traite des produits (perandren, ovocyclin, lutocyclin) qui ont vu le jour grâce à la collaboration avec Leopold Ruzicka (exactement Lavoslav Ružiãka, prix Nobel de chimie en 1939), professeur de chimie organique à l'École polytechnique de Zurich qui réussit la synthèse chimique des hormones sexuelles. Ces produits replacent la Ciba à la pointe du marché mondial des hormones sexuelles.

4 C.R. sait accompagner ses lecteurs ou lectrices qui ne sont jamais dépassés par les aspects trop techniques; l'ouvrage est accompagné de quelques illustrations, mais on regrettera l'absence d'index.

5 Paul Pasteur (université de Rouen) 